\title{
Optomechanical cooling in the non-Markovian regime
}

\author{
Wen-Zhao Zhang, ${ }^{1}$ Jiong Cheng, ${ }^{1}$ Wen-Dong $\mathrm{Li},{ }^{2}$ and Ling Zhou ${ }^{1, *}$ \\ ${ }^{1}$ School of Physics and Optoelectronic Technology, \\ Dalian University of Technology, Dalian 116024,People's Republic of China \\ ${ }^{2}$ Department of Physics, Ocean University of China, Qingdao 266100, People's Republic of China
}

\begin{abstract}
We propose a scheme in which the cooling of a mechanical resonator is achieved by exposing the optomechanical system to a non-Markovian environment. Because of the backflow from the non-Markovian environment, the phonon number can go beyond the conventional cooling limit in a Markovian environment. Utilizing the spectrum density obtained in the recent experiment [Nature Communications 6, 7606 (2015)], we show that the cooling process is highly effective in a nonMarkovian environment. The analysis of the cooling mechanism in a non-Markovian environment reveals that the non-Markovian memory effect is instrumental to the cooling process.
\end{abstract}

PACS numbers: 42.50.Wk, 07.10.Cm, 03.65.Yz, 42.50.Lc

\section{INTRODUCTION}

Recently, it has been widely recognized that optomechanical devices can be used in detecting gravity waves [1, 2], studying quantum-to-classical transitions [3], performing high precision measurements [4, 5], and processing quantum information [6, 7]. Cooling a mechanical oscillator to its quantum ground state is a prerequisite for observing quantum processes [8]. Several different kinds of systems, for example, nanomechanical systems [9 11], micromechanical systems 12, 13], and suspended mirrors in Fabry-Pérot cavities [2, 14] have been used for this purpose. For all mechanical systems, thermal noise is unavoidable unlike other noise sources that can be eliminated by using filters, screens, insulators, etc. It has been pointed out that the lowest phonon occupation number $n_{f}$ in a mechanical oscillator is limited by the phonon number $n_{\text {th }}$ of the thermal environment 15]. In order to optimize the mechanical cooling, many methods have been proposed to overcome the negative effects of the thermal environment, such as dissipative cooling [16], cooling through heat pumping [17], and cooling with mechanical modulations [18]. Up to today, studies on the mechanical-oscillator cooling have all shown that the bath heating effect of a mechanical oscillator can not be reversed easily in a typical dissipative environment.

Most recently, a non-Markovian environment for a mechanical oscillator [19] was designed and its spectrum density was measured. A non-Markovian environment exhibits the memory effect [20 22] which may play a positive role in cooling a mechanical oscillator. Therefore, it is of great importance to develop a theoretical method to solve the problems related to a nonlinear system in a non-Markovian environment.

In this paper, we introduce a non-Markovian environment for a mechanical oscillator. Taking the memory effect of the environment into consideration, we ob-

\footnotetext{
*zhlhxn@dlut.edu.cn
}

tain an analytical result for the dynamics of the phonon occupancy. We then study the optimal cooling of an optomechanical system with different spectrum densities $\mathcal{J}(\omega)$ through comparison with an optomechanical system in a Markovian environment. Furthermore, this optimal cooling can be realized with an experimental non-Ohmic spectrum density $\mathcal{J}(\omega)=C \omega^{k}$, where $C>0$ and $k \in \mathbb{R}[19]$. To understand the mechanism of non-Markovian dynamics, we analyze the equivalent energy-transport rate of the system in the cooling process. The high-temperature environment can be regarded as a freezer for the cooling of an oscillator when the nonMarkovian memory effect is included.

\section{MODEL AND HAMILTONIAN}

We consider a normal optomechanical system consisting of a cavity of frequency $\omega_{c}$ and a mechanical resonator of frequency $\omega_{m}$ as shown in Fig. 1(a). The mechanical

(a)

(b)

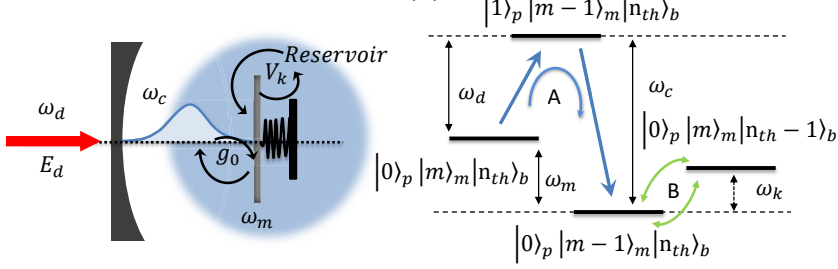

FIG. 1: (Color online) (a) Typical optomechanical system coupled to a general non-Markovian reservoir. (b) Schematic of the energy-level diagram of the cavity-optomechanical system and its environment, where $|n\rangle_{p},|m\rangle_{m}$, and $\left|n_{\mathrm{th}}\right\rangle_{b}$ represent the number states of the cavity photons, the mechanical phonons, and the reservoir phonons, respectively.

oscillator is coupled to a general non-Markovian reservoir [23, 24] that can be realized with a typical Nb pointcontact superconducting quantum-interference device in an LC circuit [25] or a high-reflectivity Bragg mirror fixed in the center of a doubly clamped $\mathrm{Si}_{3} \mathrm{~N}_{4}$ beam in vacuum 
[19]. The Hamiltonian of the system can be written as $H=H_{S}+H_{E}+H_{I}$, where

$$
\begin{aligned}
H_{S}= & \hbar \omega_{c} a^{\dagger} a+\hbar \omega_{m} b^{\dagger} b-\hbar g_{0} a^{\dagger} a\left(b^{\dagger}+b\right) \\
& +i \hbar E\left(a^{\dagger} e^{-i \omega_{d} t}-a e^{i \omega_{d} t}\right), \\
H_{E}= & \sum_{k} \hbar \omega_{k} b_{k}^{\dagger} b_{k}, \\
H_{I}= & \sum_{k} \hbar V_{k}\left(b+b^{\dagger}\right)\left(b_{k}^{\dagger}+b_{k}\right) .
\end{aligned}
$$

Here $H_{S}$ describes the cavity mode driven by a laser coupled to the mechanical resonator via radiation pressure with the coupling coefficient $g_{0}$ given by $g_{0}=$ $\left(\omega_{c} / L\right) \sqrt{h / 2 m \omega_{m}}$. In Eq. (1a), $\omega_{d}$ is the angular frequency of the laser and $E$ is the cavity driving strength given by $E \equiv 2 \sqrt{P \kappa_{e x} / \hbar \omega_{d}}$ with $P$ the input power of the laser and $\kappa_{e x}$ the input rate of the cavity. $H_{E}$ is the energy of the mechanical reservoir with $\omega_{k}$ the frequency of the $k$ th mechanical oscillator. $H_{I}$ describes the coupling between the mechanical oscillator and the reservoir with $V_{k}$ the coupling constant for the $k$ th environmental mode 24, 26].

For the convenience of studying the effects in the cooling process, we transform the Hamiltonian into the displaced oscillator representation in which the steady state of a cavity mode is the vacuum state. As illustrated in Fig. 1(b), the energy-level diagram is constructed under the sideband-cooling condition $\omega_{c}=\omega_{d}+\omega_{m}$. Kets $|0\rangle_{p}$, $|m\rangle_{m}$, and $\left|n_{\mathrm{th}}\right\rangle_{b}$ are used to dentote respectively the number states of the cavity, the mechanical oscillator, and the bath. Thus, we have anti-Stokes processes [27] in which the transition $|1\rangle_{p}|m\rangle_{m}\left|n_{\text {th }}\right\rangle_{b} \rightarrow|0\rangle_{p}|m-1\rangle_{m}\left|n_{t h}\right\rangle_{b}$ followed by the decay of the cavity photon leads to cooling. In addition, under the rotating wave approximation (RWA) with $\omega_{k} \approx \omega_{m}$, we can rewrite the Hamiltonian for the system-reservoir coupling in Eq. (1C) as $H_{I}=$ $\sum_{k} \hbar V_{k}\left(b b_{k}^{\dagger}+b^{\dagger} b_{k}\right)$ which allow the mutual energy exchange processes $|0\rangle_{p}|m\rangle_{m}\left|n_{\mathrm{th}}-1\right\rangle_{b} \leftrightarrow|0\rangle_{p}|m-1\rangle_{m}\left|n_{\mathrm{th}}\right\rangle_{b}$ to occur in which the bidirectional action of the environment can cool down or heat up the mechanical oscillator. Here the anti-Stokes-like cooling process is not obvious. We will give a detailed analysis of the cooling process in Section V to demonstrate clearly the cooling mechanism of a non-Markovian environment.

In the following calculations, we will go beyond the RWA and explore the dynamics based on the full interaction Hamiltonian. With the full Hamiltonian $H$ given in Eq. (1), the Heisenberg-Langevin equations of motion for the annihilation operators of the system are given by

$$
\begin{aligned}
\dot{a} & =-\left(i \Delta_{c}+\frac{\kappa}{2}\right) a+i g_{0} a\left(b+b^{\dagger}\right)+E+\sqrt{\kappa} a_{\mathrm{in}}, \\
\dot{b} & =-i \omega_{m} b+i g_{0} a^{\dagger} a-i \sum_{k} V_{k}\left(b_{k}+b_{k}^{\dagger}\right), \\
\dot{b_{k}} & =-i \omega_{k} b_{k}-i V_{k}\left(b+b^{\dagger}\right),
\end{aligned}
$$

where $\Delta_{c}=\omega_{c}-\omega_{d}$ and $a_{\text {in }}$ is the input noise operator of the cavity. For convenience, we take $\hbar=1$ in the remaining part of the paper. The autocorrelation function of the vacuum noise is $\left\langle a_{\mathrm{in}}(t) a_{\mathrm{in}}^{\dagger}(\tau)\right\rangle=\delta(t-\tau)$ [28]. Solving Eq. (2C) for the bath operator $b_{k}(t)$, we have

$$
\begin{aligned}
b_{k}(t)= & b_{k}(0) e^{-i \omega_{k} t} \\
& -i V_{k} \int_{0}^{t} d \tau\left[b(\tau)+b^{\dagger}(\tau)\right] e^{-i \omega_{k}(t-\tau)} .
\end{aligned}
$$

Substituting the above equation into Eq. (2b), we obtain

$$
\begin{aligned}
\dot{b}= & -i \omega_{m} b+i g_{0} a^{\dagger} a \\
& +\int_{0}^{t} d \tau f(t-\tau)\left[b(\tau)+b^{\dagger}(\tau)\right]-\xi(t),
\end{aligned}
$$

where $f(t)=2 i \sum_{k} V_{k}^{2} \sin \left(\omega_{k} t\right)=2 i \int_{0}^{\infty} d \omega \mathcal{J}(\omega) \sin (\omega t)$ with $\mathcal{J}(\omega)$ the spectral density of the reservoir. Instead of $\left[\xi(t), \xi\left(t^{\prime}\right)\right] \propto \delta\left(t^{\prime}-t\right)$ for a Markovian environment, the noise operator $\xi(t)=i \sum_{k} V_{k}\left[b_{k}(0) e^{-i \omega_{k} t}+\right.$ $\left.b_{k}^{\dagger}(0) e^{i \omega_{k} t}\right]$ has a non-local time correlation function for a non-Markovian environment. We adopt the commonly-used spectral density of the form $\mathcal{J}(\omega)=$ $\eta \omega\left(\omega / \omega_{0}\right)^{s-1} e^{-\omega / \omega_{0}}$ [29], where $\eta$ is the strength of the system-bath coupling and $\omega_{0}$ is the cut-off frequency. The exponent $s$ is a real number that determines the $\omega$ dependence of $\mathcal{J}(\omega)$ in the low-frequency region. The baths with $0<s<1, s=1$, and $s>1$ are referred to as the "sub-Ohmic", the "Ohmic", and the "super-Ohmic" baths, respectively. Here the memory kernel $f(t)$ characterizes the non-Markovian dynamics of the reservoir.

To study the dynamics of our system under the strong driving condition, we make use of the linear approximation by decomposing the operators into the classical and quantum components [15], i.e., $a \rightarrow \alpha+a$ and $b \rightarrow \beta+b$. The time evolution of the annihilation operators of the system in the Heisenberg picture is then governed by

$$
\begin{aligned}
\dot{\alpha}= & -\left(i \Delta_{c}+\frac{\kappa}{2}\right) \alpha+i g_{0} \alpha\left(\beta+\beta^{*}\right)+E, \\
\dot{\beta}= & -i \omega_{m} \beta+i g_{0}|\alpha|^{2} \\
& +\int_{0}^{t} d \tau f(t-\tau)\left[\beta(\tau)+\beta^{*}(\tau)\right], \\
\dot{a}= & -\left(i \Delta_{c}^{\prime}+\frac{\kappa}{2}\right) a+i G\left(b+b^{\dagger}\right)+\sqrt{\kappa} a_{\text {in }}, \\
\dot{b}= & -i \omega_{m} b+i\left(G a^{\dagger}+G^{*} a\right) \\
& +\int_{0}^{t} d \tau f(t-\tau)\left[b(\tau)+b^{\dagger}(\tau)\right]-\xi(t),
\end{aligned}
$$

where $\Delta_{c}^{\prime}(t)=\Delta_{c}-g_{0}\left[\beta(t)+\beta^{*}(t)\right]$ is the detuning modified by the optomechanical coupling and $G(t)=\alpha(t) g_{0}$ describes the linear coupling strength. We see that the time-dependent coefficients $\Delta_{c}^{\prime}(t)$ and $G(t)$ are determined by $\alpha(t)$ and $\beta(t)$. When the displacements of the optical and mechanical modes, $\alpha(t)$ and $\beta(t)$, are large enough, the linear approximation are satisfied. In the 
following discussions, the choice of the parameters will ensure the validity of the linear approximation.

\section{DYNAMICS OF A MECHANICAL OSCILLATOR}

In the system under study, the bath of the cavity mode is a Markovian environment to ensure that the energy of an oscillator is taken away through the cavity without reflux 27]. To examine the dynamics of a mechanical oscillator, we can eliminate the cavity mode by solving Eq. (5a) as follows

$$
\begin{aligned}
a(t)= & a(0) e^{u(t)} \\
& +\int_{0}^{t} d \tau e^{u(t-\tau)}\left\{i G(\tau)\left[b(\tau)+b^{\dagger}(\tau)\right]+\sqrt{\kappa} a_{\mathrm{in}}(\tau)\right\}
\end{aligned}
$$

where $u\left(t_{1}-t_{2}\right)=-\int_{t_{2}}^{t_{1}} d \tau\left[i \Delta^{\prime}(\tau)+\kappa / 2\right]$ describes the effect of the equivalent detuning resulting from the radiation pressure. We then have

$$
\begin{aligned}
\dot{b}= & -i \omega_{m} b+\int_{0}^{t} d \tau F(t-\tau)\left[b(\tau)+b^{\dagger}(\tau)\right] \\
& +A_{0}(t)+A_{\text {in }}(t)-\xi(t),
\end{aligned}
$$

where

$$
\begin{aligned}
F(t-\tau) & =f(t-\tau)-\left[G^{*}(t) G(\tau) e^{u(t-\tau)}-H . c .\right] \\
A_{0}(t) & =i\left[G^{*}(t) e^{u(t)} a(0)+H . c .\right] \\
A_{\text {in }}(t) & =\int_{0}^{t} d \tau i\left[\sqrt{\kappa} G^{*}(t) e^{u(t-\tau)} a_{\text {in }}(\tau)+H . c .\right] .
\end{aligned}
$$

The memory kernel $F(t)$ contains the effect of radiation pressure and $A_{0}(t)$ and $A_{\text {in }}(t)$ describe respectively the impact of the initial condition $a(0)$ and the input noise $a_{\text {in }}(t)$.

We now focus on the mechanical oscillator. In consideration of the linearity of Eq. (7), we can assume that the solution of the operator $b(t \geq 0)$ is of the form

$$
b(t)=M(t) b(0)+L^{*}(t) b^{\dagger}(0)+S(t)
$$

with the initial conditions $M(0)=1$ and $L(0)=0$. The equations for the time-dependent coefficients $L(t), M(t)$, and $S(t)$ can be found by substituting Eq. (9) into Eq. (7) and then comparing the coefficients. We have

$$
\begin{aligned}
\dot{M(}(t)= & -i \omega_{m} M(t)+\int_{0}^{t} d \tau F(t-\tau)[M(\tau)+L(\tau)] \\
\dot{L(t)}= & i \omega_{m} L(t)+\int_{0}^{t} d \tau F^{*}(t-\tau)[M(\tau)+L(\tau)] \\
\dot{S(t)}= & -i \omega_{m} S(t)+\int_{0}^{t} d \tau F(t-\tau)\left[S(\tau)+S^{\dagger}(\tau)\right] \\
& +A_{0}(t)+A_{\text {in }}(t)-\xi(t) .
\end{aligned}
$$

If $M(t)$ and $L(t)$ are known [24], the operator $S(t)$ can be completely determined through

$$
\begin{aligned}
S(t)= & \int_{0}^{t} d \tau\left[M(t-\tau)+L^{*}(t-\tau)\right] \\
& \times\left[A_{0}(\tau)+A_{\mathrm{in}}(\tau)-\xi(\tau)\right] .
\end{aligned}
$$

As shown in Eqs. (9) and (10), the solutions of the quantum parts are related to their classical parts. That is, the classical nonlinear dynamics can be manifested in the quantum properties of the system 30] even through we have made use of the linear approximation. Especially, due to the memory effects in the non-Markovian regime, the classical parts can not reach steady states as they can in the Markovian regime [16]. We will derive the dynamic solutions of classical parts by solving Eqs. (5a and (5b). Substituting the time evolution of $\alpha(t)$ and $\beta(t)$ into Eqs. (9), (10), and (11), we can obtain the time evolution of the mechanical resonator. Hence, we can thoroughly solve the problem of the non-Markovian dynamics of the phonon number without making any other approximations except the linear approximation (see Appendix A for details).

\section{SIDEBAND COOLING IN THE NON-MARKOVIAN REGIME}

We now consider the non-Markovian effect for the sideband cooling with $\Delta^{\prime}(t)=\omega_{m}$. In this case, we have $u(t)=-\left(i \omega_{m}+\kappa / 2\right) t$. To study the cooling dynamics in the non-Markovian regime, we use Eq. (9) to obtain the time evolution of the mean phonon number of the quantum part without initial system-reservoir correlations. We assume that the initial quantum number distributions are given by $\left\langle b^{\dagger}(0) b(0)\right\rangle=m_{0},\left\langle a^{\dagger}(0) a(0)\right\rangle=n_{0}$, $\left\langle a_{i n}(t) a_{i n}^{\dagger}(\tau)\right\rangle=\delta(t-\tau)$, and $\left\langle b_{k}^{\dagger}(0) b_{k}(0)\right\rangle=m_{k}$ with

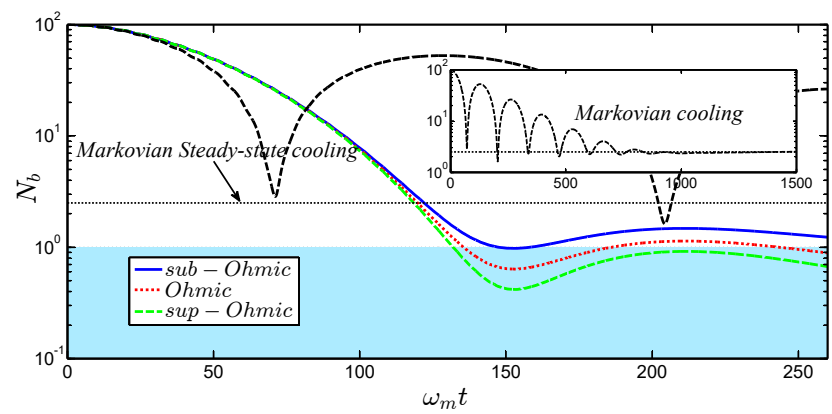

FIG. 2: (Color online) Time evolution of $N_{b}$ with a red detuning in different regimes. The Markovian dissipation $\gamma_{m}=10^{-8} \omega_{m}, s=1 / 2$ for a sub-Ohmic bath, $s=1$ for a Ohmic bath, $s=2$ for a sup-Ohmic bath, the cutoff frequency $\omega_{0} / \omega_{m}=5$, and the coupling constant $\eta=10^{-5}$ are used. The values of other parameters are $\kappa / \omega_{m}=0.05$, $g_{0} / \kappa=10^{-3}, E / \omega_{m}=300,\left|\alpha_{0}\right|=100,\left|\beta_{0}\right|=100$, and $m_{0}=10^{2}$. 
$m_{k}=1 /\left(e^{\hbar \omega_{k} / k_{B} T}-1\right)$ the photon distribution function of the reservoir. We set the mirror to be initially in thermal equilibrium with the environment with $m_{0}=1 /\left(e^{\hbar \omega_{m} / k_{B} T}-1\right)$. The time evolution of the mean phonon number $N_{b}(t)$ is then given by

$$
\begin{aligned}
N_{b}(t)= & {\left[|M(t)|^{2}+|L(t)|^{2}\right] m_{0}+|L(t)|^{2} } \\
& +\int_{0}^{t} \int_{0}^{t} d \tau_{1} d \tau_{2}\left[L\left(t-\tau_{1}\right)+M^{*}\left(t-\tau_{1}\right)\right] \\
& \times\left[L^{*}\left(t-\tau_{2}\right)+M\left(t-\tau_{2}\right)\right] \\
& \times\left[f_{1}\left(\tau_{1}, \tau_{2}\right)+f_{2}\left(\tau_{1}, \tau_{2}\right)+f_{3}\left(\tau_{1}, \tau_{2}\right)\right]
\end{aligned}
$$

where

$$
\begin{aligned}
f_{1}\left(\tau_{1}, \tau_{2}\right)= & G\left(\tau_{1}\right) G^{*}\left(\tau_{2}\right) e^{-u\left(\tau_{1}-\tau_{2}\right)} n_{0} \\
& +G^{*}\left(\tau_{1}\right) G\left(\tau_{2}\right) e^{u\left(\tau_{1}-\tau_{2}\right)}\left(n_{0}+1\right), \\
f_{2}\left(\tau_{1}, \tau_{2}\right)= & \left|G\left(\tau_{1}\right)\right|^{2}\left(1-e^{-\kappa \tau_{1}}\right), \\
f_{3}\left(\tau_{1}, \tau_{2}\right)= & \int_{0}^{\infty} \mathcal{J}(\omega) d \omega\left\{e^{-i \omega\left(\tau_{1}-\tau_{2}\right)}\right. \\
& \left.+2 \cos \omega\left(\tau_{1}-\tau_{2}\right)\left(e^{\frac{\hbar \omega}{k^{T} T}}-1\right)^{-1}\right\}
\end{aligned}
$$

in which $f_{1}$ is the contribution from the cavity photons which depends on the initial photon number $n_{0}, f_{2}$ results from the cavity input noise, and $f_{3}$ represents the effect of the oscillator bath which depends strongly on the spectral density $\mathcal{J}(\omega)$.

The time evolution of $N_{b}$ is depicted in Fig. 2 in which the initial occupation number of the oscillator is chosen to be $m_{0}=100$. For a typical suspended mirror optomechanical system with a frequency of the order of $\omega_{m}=1 \mathrm{MHz}$, the bath temperature $T \approx 1.5 \mathrm{mK}$. In comparison, the bath temperature $T \approx 1.5 \mathrm{~K}$ for a typical optical micro-resonator with $\omega_{m}=1 \mathrm{GHz}$. We compare the sideband cooling in the non-Markovian regime with several different reservoir spectral densities $\mathcal{J}(\omega)$ with that in the Markovian regime. For the Markovian regime, there exists a steady-state cooling limit $n_{f} \approx \gamma_{m} n_{\mathrm{th}}+n_{\mathrm{ce}}[15,31]$, where $n_{\mathrm{th}}$ is the equilibrium mechanical mode occupation number determined by the mechanical bath temperature and $n_{\text {ce }}$ is positive and is determined by the cavity mode and its environment.

We first consider the Markovian regime. Because of the presence of the Markovian reservoir, the mechanical oscillator is heated by its environment. To highlight the contrastive results, we choose an extremely small value for $\gamma_{m}$ with $\gamma_{m}=10^{-8} \omega_{m}$ in the Markovian regime so that the negative effect of the Markovian environment of the oscillator is negligible. See the black-dashed line in Fig. 2

In the non-Markovian regime, the phonon number is quite different from that in the Markovian regime. The time evolution of the phonon number for three kinds of spectral densities is very similar in a short period of time with $\omega_{m} t<40$. However, as time develops, the memory effect gradually sets in and the dynamics of the phonon number becomes different. Although the asymptotic

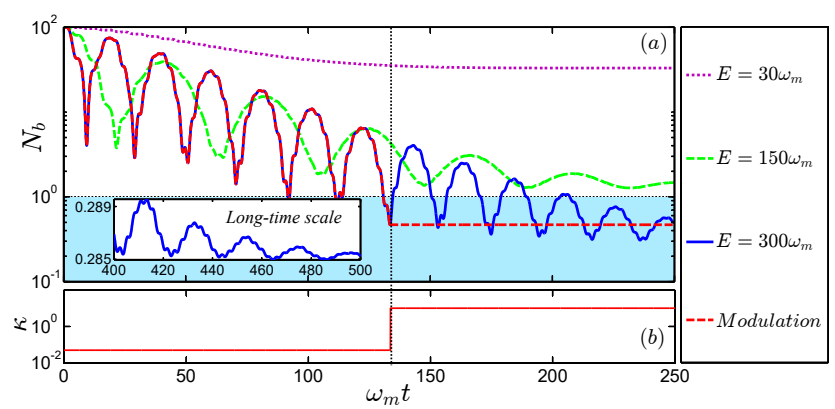

FIG. 3: (Color online) (a) Dynamics of the sideband cooling for different values of the cavity driving strength $E$ with the cavity modulation ( the red-dashed line) $E / \omega_{m}=300$. The inset shows the long-time scale with the cavity driving strength given by $E / \omega_{m}=300$. The values of other parameters are the same as in Fig. 2 (b) Modulation scheme of the cavity dissipation rate $\kappa$ for fast cooling.

steady state can not be reached in the non-Markovian regime due to the system-reservoir interaction, the mean phonon number, with small vibrations, is far below that in the Markovian regime under extreme conditions.

Recently, the spectral density of a mechanical environment was measured experimentally through the emitted light of a micro-optomechanical system [19]. The demonstration device consists of a thick layer of $\mathrm{Si}_{3} \mathrm{~N}_{4}$ with a high-reflectivity mirror pad at its center as a mechanically moving end mirror in a Fabry-Pérot cavity. The spectral density can be described by $\mathcal{J}(\omega)=C \omega^{k}$ where $C>0$ and $k=-2.30 \pm 1.05$. The concerned region of $\omega$, $\left[\omega_{\min }, \omega_{\max }\right]$ with $\omega_{\min }=885 \mathrm{kHz}$ and $\omega_{\max }=945 \mathrm{kHz}$, is centered about the mechanical resonance frequency $\omega_{m}=914 \mathrm{kHz}$ with the bandwidth given by $\Gamma \approx 0.07 \omega_{m}$. Utilizing the experimental spectral density $\mathcal{J}(\omega)=C \omega^{k}$ with $C=\eta e^{-\omega / \omega_{0}} / \omega_{0}^{k-1}, \Gamma_{m}=0.1 \omega_{m}$, and $k=-2$, we plot $N_{b}$ in Fig. 3 as a function of time.

From Fig. 3. we see that the cavity driving strength $E$ affects the cooling effect: The higher the value of $E$, the better the cooling effect. The phonon number oscillates periodically for the narrow band spectrum of the environment. After a long period of time, the mean phonon number decreases and can reach the region with $N_{b}<0.3$ for $E=300 \omega_{m}$. In consideration of the practicability and feasibility, it is always desired to speed up the cooling process. For this purpose, we can utilize the Q-switch technology by increasing suddenly the loss rate when the mean phonon number reaches the ideal value. As shown in Fig. 3 (see the solid-blue line), the phonon occupation will reach a low-excitation level in a non-steady state in a short period of time. For example, at $\omega_{m} t=133.6$, $N_{b} \approx 0.46$. At this moment, we can accelerate the stability of this low excitation state by increasing the damping rate $\kappa$ from $0.05 \omega_{m}$ to $10 \omega_{m}$. The modulation of the cavity dissipation $\kappa$ can be realized by the Q-switch technology [16]. The modulation results are shown in Fig. 3)(a) with the red-dashed line. The phonon number 
will attain a low and stable value after modulation. The modulating signal is displayed in Fig. 3(b). Furthermore, a comparison of the dashed-black line with the solid-blue line indicates that, for the same cavity driving strength $E=300 \omega_{m}$, the cooling effect for a narrow-band spectrum [19] is smaller than that for the sup-Ohmic wideband spectrum, which implies that, with the current experimental technique for a non-Markovian environment, we can obtain an optimal cooling effect.

\section{NON-MARKOVIAN COOLING MECHANISM}

As we mentioned for Fig. 1(b), a non-Markovian environment can play two opposite roles: "cooling" and "heating". In order to understand the cooling mechanism of a non-Markovian environment, we introduce the transport rate $v_{j}=d N_{j} / d t$ with $j=a$ or $b$, where $N_{a}$ and $N_{b}$ are the occupation numbers of the cavity and the mechanical oscillator, respectively. The differential equations for the mean values of the second-order moments are given in Eqs. (B1a) through (B1k in Appendix $\mathrm{B}$, in which only the beam splitter terms survive under the condition that $g_{0} / \omega_{m}, V_{k} / \omega_{m}, V_{k} / \omega_{k} \ll 1$. Then, we have $v_{a}=-v_{\kappa}+v_{c}$ [see Eq. (B1a)], where $v_{\kappa}=\kappa N_{a}$ represents the output flow of the photon energy through the cavity dissipation and $v_{c}=i\left(G\left\langle a^{\dagger} b\right\rangle-G^{*}\left\langle a b^{\dagger}\right\rangle\right)$ the input flow of the photon energy resulting from the mechanical mode due to the optomechanical interaction. The mechanical transport rate $v_{b}=-v_{c}+\Delta v$ with $\triangle v=i \sum_{k} V_{k}\left(\left\langle b^{\dagger} b_{k}\right\rangle^{*}-\left\langle b^{\dagger} b_{k}\right\rangle\right)$ [see Eq. (B1b] shows clearly that the optomechanical coupling cools the mechanical oscillator. If we can achieve $\Delta v<0$, the mechanical oscillator will be cooled further. Under the reddetuning condition with $\Delta^{\prime}(t) \approx \omega_{m}$, we have

$$
\Delta v=2 \operatorname{Im}\left(\int_{0}^{t} d \tau i\left[F_{\mathrm{th}}-\sum_{k} G V_{k}\left\langle a^{\dagger} b_{k}\right\rangle e^{i \Delta_{k}(t-\tau)}\right]\right)
$$

where $\Delta_{k}=\omega_{m}-\omega_{k}$ and $F_{\text {th }}=\int_{0}^{\infty} d \omega \mathcal{J}(\omega)\left[N_{\omega}-\right.$ $\left.N_{b}\right] e^{i\left(\omega_{m}-\omega\right)(t-\tau)}$ with $N_{\omega}=\left(e^{\hbar \omega / k_{B} T}-1\right)^{-1}$ describes the heat conduction effect from the mechanical reservoir to the oscillator. For the cooling of the mechanical oscillator, the phonon number $N_{b}$ of the mechanical oscillator is usually smaller than the phonon number $N_{\omega}$ of its environment. The integral $\int_{0}^{\infty} d \omega \mathcal{J}(\omega) e^{i\left(\omega_{m}-\omega\right)(t-\tau)}$ represents the equivalent damping rate. If $\mathcal{J}(\omega)$ is a flat spectrum, then $\int_{0}^{\infty} d \omega \mathcal{J}(\omega) e^{i\left(\omega_{m}-\omega\right)(t-\tau)}=\gamma_{m} / 2$. We can thus infer that the equivalent damping rate $\int_{0}^{\infty} d \omega \mathcal{J}(\omega) e^{i\left(\omega_{m}-\omega\right)(t-\tau)}$ is always positive. Hence, $F_{\text {th }}$ makes a positive contribution to $\Delta v$ because it is just the integral of $\mathcal{J}(\omega) e^{i\left(\omega_{m}-\omega\right)(t-\tau)}$ with the positive weight $N_{\omega}-N_{b}$. We can therefore conclude that the heat conduction of the bath has a negative effect for the cooling because of the higher thermal occupation of the environment in the cooling process.

If the second term $\sum_{k} G V_{k}\left\langle a^{\dagger} b_{k}\right\rangle e^{i \Delta_{k}(t-\tau)}$ in Eq. (14) has a positive value so as to compensate the first term, we can have $\Delta v<0$ so that we can achieve cooling. Of course, it can have a negative value or even a complex value. For a negative value, the non-Markovian backflow contributes even a worse effect for the cooling than that in a Markovian environment. In reference to the purple-dashed line with $E=30 \omega_{m}$ in Fig. 3, the value of $N_{b}$ is even higher than that in the Markovian case for $E=300 \omega_{m}$ 32 (see the purple-dashed line in Fig. 2). For a Markovian reservoir with no backflow, $\sum_{k} G V_{k}\left\langle a^{\dagger} b_{k}\right\rangle e^{i \Delta_{k}(t-\tau)}$ is zero. Therefore, the total heat conduction effect can be described as $\Delta v=\gamma_{m} n_{\text {th }}>0$. In other words, a Markovian reservoir will definitely have a negative effect on the cooling if it is desired that the temperature of the mechanical oscillator is lower than that of its environment. According to Eq. (14), if the second term is greater than the first one, then we can have a further net cooling effect. In other words, "cooling" the mechanical oscillator in the ideal case depends on the second term. The larger the second term, the better the cooling effect.

From the form of the second term, we can draw three conclusions: (1) The linearized coupling coefficient $G=$ $\alpha g_{0}$ is a controllable parameter. We can increase $\alpha$ by enhancing the cavity driving strength to achieve the ideal cooling effect, which is exactly what is demonstrated in Fig. 3. Using the parameter $\alpha$ to speed up the cooling process was discussed by Liu et al. [16], which is in consistency with Eq.(14). (2) The factor $\left\langle a^{\dagger} b_{k}\right\rangle$ indicates clearly that the backflow from the non-Markovian environment into the cavity field via the mechanical oscillator does indeed cool the mechanical oscillator with the processes $\left\langle b^{\dagger} b_{k}\right\rangle$ and $\left\langle a^{\dagger} b\right\rangle$ involved. Certainly, the Markovian environment of the cavity field is still needed since it is the final place for the lost energy of the mechanical oscillator. (3) The frequency component $\omega_{k}=\omega_{m}$ $\left(\Delta_{k}=0\right)$ yields the main contribution. If $\Delta_{k} \gg G V_{k}$, the second term in Eq. (14) is fast oscillating and makes no contribution to the cooling. In order to maintain the optimal cooling effect, the frequency of the environment should be centered about the frequency of the mechanical oscillator. Hence, when the non-Markovian memory effect is included, even the temperature of the bath is much higher than that of the phonon mode, and the environment could be also regarded as a freezer in the cooling of the oscillator.

In the review process of the paper, we noticed the new publication [34] in which the ultrafast optimal sideband cooling with a non-Markovian evolution is proposed. They optimally designed the coupling functions so as to optimize the cooling rate in both Markovian and non-Markovian environments for the cavity field as well as for the mechanical oscillator. Different from the work reported in [34], we aim to achieve lower phonon numbers in the long-time scale. Through the analysis of the cooling mechanism in a non-Markovian environment, we showed that the backflow from the non-Markovian environment of the mechanical oscillator into the cavity field 
with the further decay into the non-memory environment of the cavity field is the cause for lower phonon numbers. This conclusion coincides with [34] in which the non-Markovian dynamics in the cavity field deteriorates their cooling protocol.

\section{CONCLUSIONS}

In this paper, we put forward an environment engineering scheme for the mechanical cooling. Making use of several spectra including the one obtained in the experiment [19], we showed that the cooling effect in the present scheme is better than that in a Markovian environment. We also analyzed the cooling mechanism of a non-Markovian environment. Our analysis showed that the mechanical oscillator environment is not always detrimental to the cooling. If the environment possesses the non-Markovian memory effect, not only is the entanglement [20, 22] protected but also the mechanical cooling is optimized. A high temperature bath could be also regarded as a freezer to the cooling of the oscillator. Most importantly, with the present experiment technology, we can use a non-Markovian environment to cool a mechanical oscillator so as to go beyond the limit of a Markovian environment.

We would like to thank Mr. Wen-Lin Li and Tian-Yi Chen for helpful discussions. We also appreciate Prof. Fuxiang Han for his polishing in language. This work was supported by the NSF of China under Grant No. 11474044 .

\section{Appendix A: DYNAMICS OF A MECHANICAL OSCILLATOR}

From the expressions of $A_{0}, A_{\text {in }}$, and $\xi$, we can find that $\left\langle A_{0}(t) A_{\text {in }}(\tau)\right\rangle=0,\left\langle A_{0}(t) \xi(\tau)\right\rangle=0$, and $\left\langle A_{\text {in }}(t) \xi(\tau)\right\rangle=0$. The phonon number $N_{b}$ of the quantum part reads

$$
\begin{aligned}
\left\langle b^{\dagger}(t) b(t)\right\rangle= & |M(t)|^{2}\left\langle b^{\dagger}(0) b(0)\right\rangle+|L(t)|^{2}\left\langle b(0) b^{\dagger}(0)\right\rangle+M^{*}(t)\left\langle b^{\dagger}(0) S(t)\right\rangle+M(t)\left\langle S^{\dagger}(t) b(0)\right\rangle \\
& +L(t)\langle b(0) S(t)\rangle+L^{*}(t)\left\langle S^{\dagger}(t) b^{\dagger}(0)\right\rangle+\left\langle S^{\dagger}(t) S(t)\right\rangle,
\end{aligned}
$$

where

$$
\begin{aligned}
\left\langle b^{\dagger}(0) S(t)\right\rangle= & \int_{0}^{t} d \tau\left[M(t-\tau)-L^{*}(t-\tau)\right]\left\langle b^{\dagger}(0)\left[A_{0}(\tau)-\xi(\tau)\right]\right\rangle, \\
\langle b(0) S(t)\rangle= & \int_{0}^{t} d \tau\left[M(t-\tau)-L^{*}(t-\tau)\right]\left\langle b(0)\left[A_{0}(\tau)-\xi(\tau)\right]\right\rangle, \\
\left\langle S^{\dagger}(t) b(0)\right\rangle= & \int_{0}^{t} d \tau\left[L(t-\tau)-M^{*}(t-\tau)\right]\left\langle\left[A_{0}(\tau)-\xi(\tau)\right] b(0)\right\rangle, \\
\left\langle S^{\dagger}(t) b^{\dagger}(0)\right\rangle= & \int_{0}^{t} d \tau\left[L(t-\tau)-M^{*}(t-\tau)\right]\left\langle\left[A_{0}(\tau)-\xi(\tau)\right] b^{\dagger}(0)\right\rangle, \\
\left\langle S^{\dagger}(t) S(t)\right\rangle= & \int_{0}^{t} \int_{0}^{t}\left[L\left(t-\tau_{1}\right)-M^{*}\left(t-\tau_{1}\right)\right]\left[M\left(t-\tau_{2}\right)-L^{*}\left(t-\tau_{2}\right)\right] \\
& \times\left[\left\langle A_{0}\left(\tau_{1}\right) A_{0}\left(\tau_{2}\right)\right\rangle+\left\langle A_{\text {in }}\left(\tau_{1}\right) A_{\text {in }}\left(\tau_{2}\right)\right\rangle+\left\langle\xi\left(\tau_{1}\right) \xi\left(\tau_{2}\right)\right\rangle\right]
\end{aligned}
$$

in which the autocorrelation functions are given by

$$
\begin{aligned}
\left\langle A_{0}\left(\tau_{1}\right) A_{0}\left(\tau_{2}\right)\right\rangle & =-\left[G\left(\tau_{1}\right) G^{*}\left(\tau_{2}\right) e^{u^{*}\left(\tau_{1}\right)+u\left(\tau_{2}\right)}\left\langle a^{\dagger}(0) a(0)\right\rangle+G^{*}\left(\tau_{1}\right) G\left(\tau_{2}\right) e^{u\left(\tau_{1}\right)+u^{*}\left(\tau_{2}\right)}\left\langle a(0) a^{\dagger}(0)\right\rangle\right], \\
\left\langle A_{i n}\left(\tau_{1}\right) A_{i n}\left(\tau_{2}\right)\right\rangle & =-\left|G\left(\tau_{1}\right)\right|^{2} \kappa e^{u\left(\tau_{1}\right)+u^{*}\left(\tau_{1}\right)} \int_{0}^{\tau_{1}} d \tau e^{-u(\tau)-u^{*}(\tau)}\left\langle a_{i n}(\tau) a_{i n}^{\dagger}(\tau)\right\rangle, \\
\left\langle\xi\left(\tau_{1}\right) \xi\left(\tau_{2}\right)\right\rangle & =-\sum_{k} V_{k}^{2}\left[e^{-i \omega_{k}\left(\tau_{1}-\tau_{2}\right)}\left\langle b_{k}(0) b_{k}^{\dagger}(0)\right\rangle+e^{i \omega_{k}\left(\tau_{1}-\tau_{2}\right)}\left\langle b_{k}^{\dagger}(0) b_{k}(0)\right\rangle\right] .
\end{aligned}
$$


The cross-correlation functions are given by

$$
\begin{aligned}
\left\langle b^{\dagger}(0) A_{0}(t)\right\rangle & =i\left[G^{*}(t) e^{u(t)}\left\langle b^{\dagger}(0) a(0)\right\rangle+G(t) e^{u^{*}(t)}\langle a(0) b(0)\rangle^{*}\right], \\
\left\langle b^{\dagger}(0) \xi(t)\right\rangle & =i \sum_{k} V_{k}\left[e^{-i \omega_{k} t}\left\langle b_{k}(0) b^{\dagger}(0)\right\rangle^{*}+e^{i \omega_{k} t}\left\langle b_{k}(0) b(0)\right\rangle^{*}\right], \\
\left\langle b(0) A_{0}(t)\right\rangle & =i\left[G^{*}(t) e^{u(t)}\langle b(0) a(0)\rangle+G(t) e^{u^{*}(t)}\left\langle a(0) b^{\dagger}(0)\right\rangle^{*}\right], \\
\langle b(0) \xi(t)\rangle & =i \sum_{k} V_{k}\left[e^{-i \omega_{k} t}\left\langle b(0) b_{k}(0)\right\rangle+e^{i \omega_{k} t}\left\langle b(0) b_{k}^{\dagger}(0)\right\rangle\right], \\
\left\langle A_{0}(t) b(0)\right\rangle & =i\left[G^{*}(t) e^{u(t)}\langle a(0) b(0)\rangle+G(t) e^{u^{*}(t)}\left\langle b^{\dagger}(0) a(0)\right\rangle^{*}\right], \\
\langle\xi(t) b(0)\rangle & =i \sum_{k} V_{k}\left[e^{-i \omega_{k} t}\left\langle b_{k}(0) b(0)\right\rangle+e^{i \omega_{k} t}\left\langle b_{k}^{\dagger}(0) b(0)\right\rangle\right], \\
\left\langle A_{0}(t) b^{\dagger}(0)\right\rangle & =i\left[G^{*}(t) e^{u(t)}\left\langle a(0) b^{\dagger}(0)\right\rangle+G(t) e^{u^{*}(t)}\langle b(0) a(0)\rangle^{*}\right], \\
\left\langle\xi(t) b^{\dagger}(0)\right\rangle & =i \sum_{k} V_{k}\left[e^{-i \omega_{k} t}\left\langle b(0) b_{k}^{\dagger}(0)\right\rangle^{*}+e^{i \omega_{k} t}\left\langle b(0) b_{k}(0)\right\rangle^{*}\right] .
\end{aligned}
$$

The solution to Eq. A1 depends on the initial values of the photon-phonon correlation functions $\langle a(0) b(0)\rangle$, $\langle b(0) a(0)\rangle,\left\langle a(0) b^{\dagger}(0)\right\rangle$, and $\left\langle b^{\dagger}(0) a(0)\right\rangle$ and on the initial values of the mirror-reservoir correlation functions $\left\langle b(0) b_{k}(0)\right\rangle$, $\left\langle b_{k}(0) b(0)\right\rangle,\left\langle b(0) b_{k}^{\dagger}(0)\right\rangle$, and $\left\langle b_{k}^{\dagger}(0) b(0)\right\rangle$. Equation (A1) can be solved by making use of Eq. (10) and the initial values of the system-reservoir correlation functions.

\section{Appendix B: DYNAMICS OF THE SYSTEM}

To understand clearly the role played by the environment in the cooling process, we study the time evolution of the photon and phonon numbers by making use of the original Heisenberg-Langevin equations. Applying the linear approximation, we can simplify the dynamical equations. Here, the system is surrounded by a large environment whose occupation number $N_{k}$ can be regarded as a constant. The simplified dynamical equations are given by

$$
\begin{aligned}
& \frac{d\left\langle N_{a}\right\rangle}{d t}=-\kappa N_{a}+i\left(G\left\langle a^{\dagger} b\right\rangle-G^{*}\left\langle a^{\dagger} b\right\rangle^{*}+G\langle a b\rangle^{*}-G^{*}\langle a b\rangle\right), \\
& \frac{d\left\langle N_{b}\right\rangle}{d t}=-i\left(G\left\langle a^{\dagger} b\right\rangle-G^{*}\left\langle a^{\dagger} b\right\rangle^{*}+G\langle a b\rangle^{*}-G^{*}\langle a b\rangle\right)+i \sum_{k} V_{k}\left(\left\langle b b_{k}\right\rangle-\left\langle b b_{k}\right\rangle^{*}+\left\langle b^{\dagger} b_{k}\right\rangle^{*}-\left\langle b^{\dagger} b_{k}\right\rangle\right), \\
& \frac{d\left\langle a^{\dagger} b\right\rangle}{d t}=-\left[i\left(\omega_{m}-\Delta_{c}^{\prime}\right)+\kappa / 2\right]\left\langle a^{\dagger} b\right\rangle-i\left(G^{*}\left\langle b^{2}\right\rangle+G^{*} N_{b}-G^{*} N_{a}-G\left\langle a^{2}\right\rangle^{*}\right)-i \sum_{k} V_{k}\left(\left\langle a^{\dagger} b_{k}\right\rangle+\left\langle a b_{k}\right\rangle^{*}\right), \\
& \frac{d\left\langle a^{\dagger} b_{k}\right\rangle}{d t}=-\left[i\left(\omega_{k}-\Delta_{c}^{\prime}\right)+\kappa / 2\right]\left\langle a^{\dagger} b_{k}\right\rangle-i G^{*}\left(\left\langle b b_{k}\right\rangle+\left\langle b^{\dagger} b_{k}\right\rangle\right)-i V_{k}\left(\left\langle a^{\dagger} b\right\rangle+\langle a b\rangle^{*}\right), \\
& \frac{d\left\langle b^{\dagger} b_{k}\right\rangle}{d t}=i\left(\omega_{m}-\omega_{k}\right)\left\langle b^{\dagger} b_{k}\right\rangle-i\left(G^{*}\left\langle a b_{k}\right\rangle+G\left\langle a^{\dagger} b_{k}\right\rangle\right)+i V_{k}\left(\left\langle b_{k}^{2}\right\rangle+N_{k}-N_{b}-\left\langle b^{2}\right\rangle^{*}\right), \\
& \frac{d\langle a b\rangle}{d t}=-\left[i\left(\Delta_{c}^{\prime}+\omega_{m}\right)+\kappa / 2\right]\langle a b\rangle+i\left(G\left\langle b^{2}\right\rangle+G N_{b}+G^{*}\left\langle a^{2}\right\rangle+G\left\langle a a^{\dagger}\right\rangle\right)-i \sum_{k} V_{k}\left(\left\langle a b_{k}\right\rangle+\left\langle a^{\dagger} b_{k}\right\rangle^{*}\right), \\
& \frac{d\left\langle a b_{k}\right\rangle}{d t}=-\left[i\left(\Delta_{c}^{\prime}+\omega_{k}\right)+\kappa / 2\right]\left\langle a b_{k}\right\rangle+i G\left(\left\langle b b_{k}\right\rangle+\left\langle b^{\dagger} b_{k}\right\rangle\right)-i V_{k}\left(\langle a b\rangle+\left\langle a^{\dagger} b\right\rangle^{*}\right), \\
& \frac{d\left\langle b b_{k}\right\rangle}{d t}=-i\left(\omega_{m}+\omega_{k}\right)\left\langle b b_{k}\right\rangle+i\left(G^{*}\left\langle a b_{k}\right\rangle+G\left\langle a^{\dagger} b_{k}\right\rangle\right)-i V_{k}\left(\left\langle b_{k}^{2}\right\rangle+N_{k}+\left\langle b^{2}\right\rangle+\left\langle b b^{\dagger}\right\rangle\right), \\
& \frac{d\left\langle a^{2}\right\rangle}{d t}=-\left(2 i \Delta_{c}^{\prime}+\kappa\right)\left\langle a^{2}\right\rangle+2 i G\left(\langle a b\rangle+\left\langle a^{\dagger} b\right\rangle^{*}\right), \\
& \frac{d\left\langle b^{2}\right\rangle}{d t}=-2 i \omega_{m}\left\langle b^{2}\right\rangle+2 i\left(G^{*}\langle a b\rangle+G\left\langle a^{\dagger} b\right\rangle\right)-2 i \sum_{k} V_{k}\left(\left\langle b b_{k}\right\rangle+\left\langle b^{\dagger} b_{k}\right\rangle^{*}\right), \\
& \frac{d\left\langle b_{k}^{2}\right\rangle}{d t}=-2 i \omega_{k}\left\langle b_{k}^{2}\right\rangle-2 i V_{k}\left(\left\langle b b_{k}\right\rangle+\left\langle b^{\dagger} b_{k}\right\rangle\right) .
\end{aligned}
$$


The equivalent transport rates of the cavity and the mechanical modes are $v_{a}=d N_{a} / d t$ and $v_{b}=d N_{b} / d t$, respectively. We set the laser to be red detuned with $\Delta^{\prime}(t)=\omega_{m}$ for which the beam splitter interaction is in resonance. Under the weak-coupling condition that $g_{0} / \omega_{m}, V_{k} / \omega_{m}, V_{k} / \omega_{k} \ll 1$, we can identify the fast oscillating terms with the corresponding evolution equations given in Eqs. (B1f) through (B1k). We can then adiabatically eliminate these fast oscillating terms in Eqs. (B1 ) through (B1). In Eq. (B1 $)$, the first term contains the transport rate through the dissipation rate $\kappa$ and the second term contains the transport rate $v_{c}$ with $v_{c}=i\left(G\left\langle a^{\dagger} b\right\rangle-G^{*}\left\langle a b^{\dagger}\right\rangle\right)$ from the mechanical mode through the optomechanical coupling. In Eq. (B1b), the first term contains the output flow $v_{c}$ through the optomechanical coupling and the second term contains the transport rate $\triangle v=i \sum_{k} V_{k}\left(\left\langle b^{\dagger} b_{k}\right\rangle^{*}-\left\langle b^{\dagger} b_{k}\right\rangle\right)$ due to the oscillator-reservoir interaction. Combining Eqs. (B1b) and (B1) we have

$$
\triangle v=2 V_{k} \operatorname{Im}\left(\int_{0}^{t} d \tau i\left[\sum_{k} e^{i \Delta_{k}(t-\tau)} V_{k}^{2}\left(N_{k}-N_{b}\right)-\sum_{k} e^{i \Delta_{k}(t-\tau)} G V_{k}\left\langle a^{\dagger} b_{k}\right\rangle\right]\right),
$$

where $\Delta_{k}=\omega_{m}-\omega_{k}$ and $\sum_{k} e^{i \Delta_{k}(t-\tau)} V_{k}^{2}\left(N_{k}-N_{b}\right)=\int_{0}^{\infty} d \omega \mathcal{J}(\omega)\left[\left(e^{\hbar \omega / k_{B} T}-1\right)^{-1}-N_{b}\right] e^{i\left(\omega_{m}-\omega\right)(t-\tau)} \operatorname{describes}$ the heat conduction effect from the mechanical reservoir to the oscillator. The second term in the integrand in Eq. (B2) that contains $\left\langle a^{\dagger} b_{k}\right\rangle$ describes the non-Markovian memory effect from the cavity dynamics through the optomechanical interaction. The two terms in the integrand in Eq. (B2) make opposite contributions in the cooling process. When $\triangle v<0$, the reservoir will exhibit a "cooling" effect to the mechanical oscillator. We can achieve this effect by increasing the linearized coupling rate $G$. We also notice that, if $\Delta_{k} \gg G V_{k}$, the second term then becomes fast oscillating and hence makes no contribution to the cooling. To maintain the cooling effect, we should have $\Delta_{k} \ll G V_{k}$. That is, the frequency of the environment should be centered about the frequency of the mechanical oscillator. Therefore, when the system exhibits non-equilibrium dynamics [33], it is possible that the temperature of the mechanical oscillator is much lower than that of the environment.

[1] Y. Ma, S. L. Danilishin, C. Zhao, H. Miao, W. Z. Korth, Y. Chen, R. L. Ward, and D. G. Blair, Phys. Rev. Lett. 113, 151102 (2014).

[2] B. Abbott, R. Abbott, R. Adhikari, P. Ajith, B. Allen, G. Allen, R. Amin, S. B. Anderson, W. G. Anderson, M. A. Arain, et al., New Journal of Physics 11, 073032 (2009).

[3] R. Ghobadi, S. Kumar, B. Pepper, D. Bouwmeester, A. I. Lvovsky, and C. Simon, Phys. Rev. Lett. 112, 080503 (2014).

[4] K. Zhang, F. Bariani, Y. Dong, W. Zhang, and P. Meystre, Phys. Rev. Lett. 114, 113601 (2015).

[5] S. Barzanjeh, S. Guha, C. Weedbrook, D. Vitali, J. H. Shapiro, and S. Pirandola, Phys. Rev. Lett. 114, 080503 (2015).

[6] W.-Z. Zhang, J. Cheng, J.-Y. Liu, and L. Zhou, Phys. Rev. A 91, 063836 (2015).

[7] W. Li, C. Li, and H. Song, Journal of Physics B: Atomic, Molecular and Optical Physics 48, 035503 (2015).

[8] J. Jing, L.-A. Wu, M. Byrd, J. Q. You, T. Yu, and Z.-M. Wang, Phys. Rev. Lett. 114, 190502 (2015).

[9] A. Jöckel, A. Faber, T. Kampschulte, M. Korppi, M. T. Rakher, and P. Treutlein, Nature Nanotechnology 10, 55 (2014).

[10] G. A. T. Pender, P. F. Barker, F. Marquardt, J. Millen, and T. S. Monteiro, Phys. Rev. A 85, 021802 (2012).

[11] T. Bagci, a. Simonsen, S. Schmid, L. G. Villanueva, E. Zeuthen, J. Appel, J. M. Taylor, a. Sørensen, K. Usami, a. Schliesser, et al., Nature 507, 81 (2014).

[12] M. Yuan, V. Singh, Y. M. Blanter, and G. A. Steele, Nature Communications 6, 8491 (2015).

[13] J. D. Teufel, T. Donner, D. Li, J. W. Harlow, M. S.
Allman, K. Cicak, a. J. Sirois, J. D. Whittaker, K. W. Lehnert, and R. W. Simmonds, Nature 475, 359 (2011).

[14] H. J. Eerkens, F. M. Buters, M. J. Weaver, B. Pepper, G. Welker, K. Heeck, P. Sonin, S. de Man, and D. Bouwmeester, Optics Express 23, 8014 (2015).

[15] M. Aspelmeyer, T. J. Kippenberg, and F. Marquardt, Rev. Mod. Phys. 86, 1391 (2014).

[16] Y.-C. Liu, Y.-F. Xiao, X. Luan, and C. W. Wong, Phys. Rev. Lett. 110, 153606 (2013).

[17] Y. Dong, F. Bariani, and P. Meystre, Phys. Rev. Lett. 115, 223602 (2015).

[18] M. Bienert and P. Barberis-Blostein, Phys. Rev. A 91, 023818 (2015).

[19] S. Gröblacher, A. Trubarov, N. Prigge, G. D. Cole, M. Aspelmeyer, and J. Eisert, Nature Communications 6, 7606 (2015).

[20] J. Cheng, W.-Z. Zhang, Y. Han, and L. Zhou, Phys. Rev. A 91, 022328 (2015).

[21] J. Jing and T. Yu, Phys. Rev. Lett. 105, 240403 (2010).

[22] D. M. Reich, N. Katz, and C. P. Koch, Scientific Reports 5, 12430 (2015).

[23] H.-T. Tan and W.-M. Zhang, Phys. Rev. A 83, 032102 (2011).

[24] K. W. Chang and C. K. Law, Phys. Rev. A 81, 052105 (2010).

[25] A. J. Leggett, Phys. Rev. B 30, 1208 (1984).

[26] V. Giovannetti and D. Vitali, Phys. Rev. A 63, 023812 (2001).

[27] I. Wilson-Rae, N. Nooshi, W. Zwerger, and T. J. Kippenberg, Phys. Rev. Lett. 99, 093901 (2007).

[28] C. W. Gardiner and P. Zoller, Quantum Noise (Springer- 
Verlag, Berlin, 2000).

[29] A. J. Leggett, S. Chakravarty, A. T. Dorsey, M. P. A. Fisher, A. Garg, and W. Zwerger, Rev. Mod. Phys. 59, 1 (1987).

[30] G. Wang, L. Huang, Y.-C. Lai, and C. Grebogi, Phys. Rev. Lett. 112, 110406 (2014).

[31] F. Marquardt, J. P. Chen, A. A. Clerk, and S. M. Girvin, Phys. Rev. Lett. 99, 093902 (2007).
[32] In Markovian regime, the the quantum cooling limit $N_{\text {limit }} \propto|G|^{2}$. The cooling limit for smaller $E=30 \omega_{m}$ is lower than that for $E=300 \omega_{m}$.

[33] J. Cheng, W.-Z. Zhang, L. Zhou, and W. Zhang, Sci. Rep. 6, 23678 (2016).

[34] J. F. Triana, A. F. Estrada, and L. A. Pachon, Phys. Rev. Lett. 116, 183602 (2016). 